\title{
Comparison of Analytical and Simulation Approaches for Assessing Robustness of Reliability for Water Distribution Systems
}

Jinhui Huang and Edward A. McBean

Characterizations of reliability for water distribution systems have been studied for decades in terms of mechanical reliability, hydraulic reliability and topology reliability. The various methods, described as either simulation approaches or analytical approaches, each have both advantages and limitations but used in combination, provide municipal engineers the tools to evaluate the performance reliability for existing systems, and indications how to improve their networks to achieve more reliable and robust reliability. The analytical approach is shown to provide insight into the water distribution system and gives a static view of the reliability in terms of topology, while the simulation approach offers the opportunity to investigate real world scenarios and evaluate the consequence of mechanical failure in terms of pressure and demand.

To evaluate the hydraulic reliability of a water distribution system against mechanical failure, Monte-Carlo Simulation coupled with EPANET Toolkit was employed. The analytical approach employed the decomposition method to evaluate the connectivity of the entire distribution system. An improved boundary set identification method was developed, allowing the analytical approach to become feasible in application to real systems.

Huang, J. and E. McBean. 2006. "Comparison of Analytical and Simulation Approaches for Assessing Robustness of Reliability for Water Distribution Systems." Journal of Water Management Modeling R225-11. doi: 10.14796/JWMM.R225-11.

(C) CHI 2006 www.chijournal.org ISSN: 2292-6062 (Formerly in Intelligent Modeling of Urban Water Systems. ISBN: 0-9736716-2-9) 
Case studies were used to examine the feasibility and applicability of the simulation approach and the analytical approach and to evaluate their efficiency and accuracy.

\subsection{Introduction}

The needs for reliable water supply infrastructure to meet existing and changing societal water demands are apparent. The designer of the infrastructure system thus must understand the reliability of the system in response to, for example, urban growth, water demands, and aging infrastructure. However, quantifying the reliability (resilience against failure) of a water supply system continues to be a challenging problem, and no universal reliability methodology is available (Mays, 1996; Huang, McBean and James, 2003).

While issues regarding alternative approaches exist, research characterizing the reliability of components of a water infrastructure system has received significant attention. Models characterizing reliability, defined as the probability that the system operates successfully over a specified time interval, include models for reliability related to pipe burst, and valve failure (e.g. Shamir and Howard, 1979; Goulter et al., 1986; Su et al., 1987; Goulter and Kazemi, 1988; Kleiner and Rajani, 2001; Hosoi and Kido, 2001; and Babovic et al., 2002). From the reliability of individual components, the reliability of the overall water distribution infrastructure system has been characterized using both (i) analytical approaches and (ii) simulation approaches.

Analytical approaches assess reliability performance based on a network's fundamental parameters (e.g. number of links connected to each node, size of pipes, location of nodes, and demands), while simulation approaches evaluate the network's performance under a range of scenarios including those of potential component failure phenomena. Although each approach has both advantages and limitations, the two approaches used in combination, can be effective at assessing the adequacy of water distribution system reliability. The intent of this chapter is to compare the performance of simulation and analytical approaches and provide guidelines for their individual use. The chapter focuses on the use of 'connectivity' and examines the feasibility to use the connectivity as an approach to characterize the reliability for water distribution infrastructure. 


\subsection{Simulation Approaches to Reliability Characterization}

Simulation approaches have been employed in hydraulic reliability analyses for decades (e.g. Hobbs and Beim, 1988; Wagner et al., 1988b; Xu and Goulter, 1998; Tanyimboh et al., 1999; Ostfeld 2001; Ostfeld et al., 2002; Kalungi, et al., 2003; Tolson et al., 2004). "Hydraulic reliability", the reliability in meeting demand and pressure constraints has long been employed as a measure of system reliability. While hydraulic reliability is necessary (e.g. to ensure reliable performance in response to firefighting flow demands plus maximum daily demands), quantifying hydraulic reliability from component reliability is equally valuable. Specifically, at each node, a reliable system needs to maintain pressure within a specified $\mathrm{min} / \mathrm{max}$ range assigned from service performance requirements (e.g. between $138 \mathrm{kN} / \mathrm{m} 2(20 \mathrm{psi})$ and $552 \mathrm{kN} / \mathrm{m} 2$ (80 psi) under normal daily demand (Shinstine et al., 2002)).

Previous work (Goulter and Coals 1986; Su et al. 1987) proposed the use of a discrete relationship between reliability and pressure: $\mathrm{HA}_{j}=1$ for $\mathrm{P}_{j} \geq$ $\mathrm{P}_{\mathrm{R}}$, whereas $\mathrm{HA}_{\mathrm{j}}=0$ for $\mathrm{P}_{\mathrm{j}} \leq \mathrm{PR}$, where $\mathrm{HA}_{\mathrm{j}}=$ hydraulic reliability of node $\mathrm{j} ; \mathrm{P}_{\mathrm{j}}=$ pressure at node $\mathrm{j}$; and $\mathrm{PR}=$ required minimum pressure. Herein, we adopt this approach for both nodal pressures and nodal demands. The hydraulic reliability is thus defined as the demand reliability and the pressure reliability respectively, as outlined in (11.1) and (11.2).

- Demand Reliability $\left(\mathrm{R}_{\mathrm{Di}}\right)$ :

- $\mathrm{R}_{\mathrm{Di}}=0$, when $0 \leq \mathrm{D}_{\text {real }}<\mathrm{D}_{\text {Base }}$

- $\mathrm{R}_{\mathrm{Di}}=1$, when $\mathrm{D}_{\text {real }}>\mathrm{D}_{\text {Base }}$

- Pressure Reliability $\left(\mathrm{P}_{\mathrm{R}}\right)$

where:

- $\mathrm{R}_{\mathrm{Pi}_{\mathrm{i}}}=0$, when $\mathrm{P}<\mathrm{Pmin}$, or $\mathrm{P}>\mathrm{Pmax}$

- $\mathrm{R}_{\mathrm{Pi}}=1$, when Pmin $\leq \mathrm{P} \leq \mathrm{P} \max$

$\mathrm{i}=1,2, \ldots \mathrm{N} ; \mathrm{N}$ is the number of supply nodes in the distribution system

$\mathrm{D}_{\text {real }}=$ the real demand at a node, which could be the computed demand by the hydraulic model or the measured demand at a monitoring station;

$D_{\text {Base }}=$ the specified water demand at a node, which is the demand need defined by land use and time; 


$$
\begin{aligned}
\mathrm{P} & =\text { the pressure at a node and; } \\
\mathrm{P}_{\min } \text { and } \mathrm{P}_{\max }= & \text { the required minimum/maximum pressures } \\
& \text { respectively }
\end{aligned}
$$

Once the reliability in terms of meeting the demands and pressures for each node has been determined, the system reliability can thus be determined from (11.3) and (11.4).

Demand Reliability

$$
\left(\sum_{i=1}^{N}\left(R_{i} \times \text { BaseDemand }_{i}\right)\right) / \text { TotalBaseDemand }
$$

Pressure Reliability

$$
\left(\sum_{i=1}^{N} R_{i}\right) / N
$$

where:

$\mathrm{R}_{\mathrm{i}}=$ Reliability for Each Nodes

Monte Carlo method was used in simulation approach to characterize the system reliability. The procedure to run a Monte-Carlo simulation is summarized in Table 11.1.

Table 11.1 Sequence of calculation steps for the simulation analyses.

1) Assign each link a reliability rate

2) Randomly generate the operational mode (operating or closed) of each link based on its reliability rate

3) Set a link to be opened if it is in operation mode or set a link to be closed if it is in failure mode

4) Run a hydraulic simulation model (EPANET) and determine the demands and pressures for each node

5) From the computed pressures and demands, the reliability of each node for each trial is evaluated using[1] and [2]

6) Repeat (2) through (5) for a number of trials, determine the average reliability of each node and compute the reliability of the system is obtained from [3] and [4].

EPANET was employed to perform hydraulic simulations described herein. All the pipes, valves and pumps were treated as links in EPANET (Rossman, 2000); only link failures are considered in this study. In other words, all 
nodes were considered to be perfectly reliable. This assumption also applied to the analytical approach; to allow the results from the simulation approach and analytical approach to be comparable.

\subsection{Analytical Approach to Reliability Characterization}

Goulter (1988) and Jacobs and Goulter (1989) first formally proposed to use graph theory in assessing water distribution system reliability. Since then, graph theory has been applied extensively in analytical approaches (e.g. Mays, 2000). Three approaches in graph theory have been introduced to study the reliability of water distribution systems, namely:

1. Reachability - Reachability is defined as the probability that a specified demand node is connected to a source node (i.e. there is a pathway by which water can be delivered from a source to an individual node, and hence, is a measure of the reliability of an individual node (e.g. Wagner et al., 1988, Quimpo and Shamsi, 1991; Yang et al., 1996; Hosoi et al. 2001).

2. Connectivity - Connectivity is defined as the probability that all nodes are connected to each other by at least one pathway and hence is a measure of the reliability of the entire system. (e.g. Wagner et al., 1988a).

3. Minimum cut-set - Minimum cut-set is a set of links that, when removed from a network, one or more nodes are isolated from the remaining network (e.g. Su et al. 1987; Jacobs and Goulter, 1988; Shinstine, et al. 2002).

Defining reachability as the probability that at least one demand node is connected to a particular source node, the reliability of the distribution system can be evaluated by integrating over all individual reachabilities. However, the reachability of an individual node is not independent from the reachabilities of its upstream node(s). As a consequence, the reachability approach for an entire system double-counts the effects of some links, and overestimates the reliability result. The same deficiency exists regarding the minimum cut-set approach. The minimum cut set approach is focused on individual nodes rather than the entire network, it thus has the same 
deficiency as reachability approach. Additionally, due to computational feasibility concerns, the identification of a minimum cut set usually uses a first-order or second-order approach, the result is thus only an approximation to the true value. In short, the reliability of the entire network in terms of reachability and cut-set only give a rough estimation, indicating the connectivity approach is a better characterization of the reliability for the entire network.

While having the above-noted limitations, for computational reasons, most research has focused on the reachability and minimum cut-set methods. The 'connectivity' approach has received less attention in the literature since it first proposed by Wagner et al. (1988a). Wagner et al. (1988a) also reported that the connectivity method at that time could only solve moderate-size networks, e.g. 10-25 demand nodes and 10-50 links. These restrictions limited the use of connectivity in reliability evaluations for water distribution systems. However, as detailed below, an alternative implementation of the connectivity method relaxes this limitation.

\subsubsection{Network Connectivity Approach}

Network connectivity has been studied in the fields of internet communications and operational research for several decades. In terms of topology, all networks can be specified as either series-parallel reducible networks, or general irreducible networks. Series-parallel networks are networks which can be reduced into a dendritic network (without loops), while a general irreducible network cannot be reduced into a tree-like network; in words, a loop-free network cannot be found after a series of reductions. For the connectivity formulation, the dendritic network is the easiest network to solve, while in contrast, the general irreducible network has been identified to be a NP-hard problem; in words, a very complicated problem which may not be solved in an affordable time by computer. Wagner et al. (1988a) used Rosenthal's decomposition method, which was developed for electric communication applications, to formulate the connectivity model of water distribution systems. However, only two very simple hypothetical systems were examined. One consisted of 10 nodes and 12 links. Another consisted of 10 nodes and 14 links. The major problem in adopting Graph Theory to the reliability assessment of water distributions system is that these systems usually consist of large numbers of nodes and links. The increased number of nodes and links increases computational time dramatically. Based on the experience of $\mathrm{Su}$ et al. (1987), the computation time required for "optimization" increased from 1157 s to $12030 \mathrm{~s}$ on a CDC CYBER machine 
when a 14-links-network became a 17-link-network and hence this formulation rapidly becomes infeasible in application.

In accordance with graph theory, distribution systems with loops are modeled by an undirected graph G (V, E), whose vertices (nodes) and edges (pipes between each node pairs) are subject to statistically independent failures, with specified failure probabilities. $\mathrm{V}$ is the entire set of vertices in $\mathrm{G}$ and $\mathrm{E}$ is the entire set of 'edges' in $\mathrm{G}$. If $\mathrm{p}_{\mathrm{e}}$ denotes the reliability of edge $\mathrm{e}=(\mathrm{u}, \mathrm{v}), \mathrm{q}_{\mathrm{e}}$ is the failure rate of edge $\mathrm{e}, \mathrm{q}_{\mathrm{e}}$ equals to $1-\mathrm{p}_{\mathrm{e}}$, where $\mathrm{u} \neq \mathrm{v}$; and $\mathrm{u} \in \mathrm{V}, \mathrm{v} \in \mathrm{V} ; \mathrm{e} \in \mathrm{E}$. If a possible event identified to have $\mathrm{m}$ links up (is functioning) and $\mathrm{n}$ links down (in failure mode) in the system, then the probability of this event becomes

$$
\prod_{i=1}^{m} p_{i} \times \prod_{j=1}^{n} q_{j}
$$

The reliability of the entire system is the sum of all possible events which ensure the reliability of the system, subject to specified criteria. For a network with $\mathrm{N}$ links, the total number of outcomes is $2^{\mathrm{N}}$. Therefore, when the number of pipes, $\mathrm{N}$, is equal to 100 , the number of possible outcomes is $2^{100}=1.27 \times 10^{30}$ demonstrating infeasibility for enumeration of all possible events to get the reliability characterization. Programmable, efficient methods are needed.

\section{Decomposition Method}

If a graph $\mathrm{G}$ can be decomposed into two sub-graphs $\mathrm{A}=\left(\mathrm{V}^{\prime}, \mathrm{E}^{\prime}\right)$ and $\mathrm{B}\left(\mathrm{V}^{\prime}\right.$, $\mathrm{E}$ "), as per Figure 11.1, $\mathrm{A}$ and $\mathrm{B}$ are complementary and only have an articulation vertex in common. Thus $\mathrm{R}(\mathrm{G})=\mathrm{R}(\mathrm{A}) * \mathrm{R}(\mathrm{B})$.

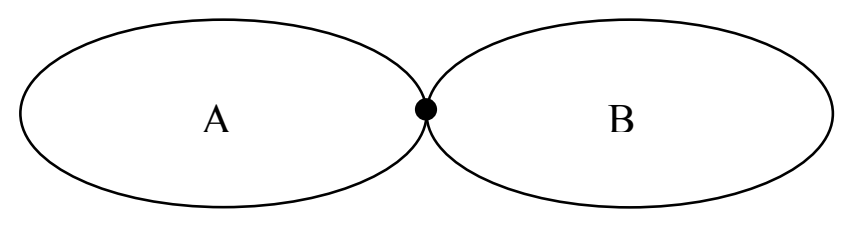

Figure 11.1 An articulation vertex .

While A and B have vertices in common, as illustrated in Figure 11.2, C is their boundary set with $A \cap B=C$. The $R(G)$ depends on the value of $\mathrm{R}(\mathrm{A})$ and $\mathrm{R}(\mathrm{B})$, as well as the connectivity of $\mathrm{C}$ and how the nodes in the boundary/overlap set $\mathrm{C}$ connect with $\mathrm{A}$ and $\mathrm{B}$. 
Rosenthal (1977) solved the connectivity problem from a simple graph, to a complicated graph. For example, in Figure 11.3, the graph can be separated into subgraph A and subgraph B. The links in B is shown in solid line and the links in $\mathrm{A}$ is presented in dashed line. A and $\mathrm{B}$ have three nodes in common. They are nodes 2,6 and 10 . The boundary set is defined as $\mathrm{C}=$ $\{2,6,10\}$, and $\mathrm{A}$ and $\mathrm{B}$ with $\mathrm{C}$ are 3 -subnets according to Rosenthal.

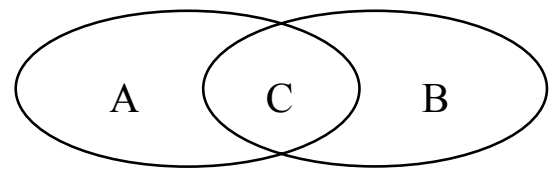

Figure 11.2 A boundary set.

There are five connection states (also termed classes by Rosenthal, 1977) via A or B. They are [2 6 10]; [2 10][6]; [2 6][10]; [2][6 10]; [2][10][6]. The connectivity of the graph thus can be separated into several sub problems: the connectivity of sub network A and B, and the probability of classes in $\mathrm{C}$.

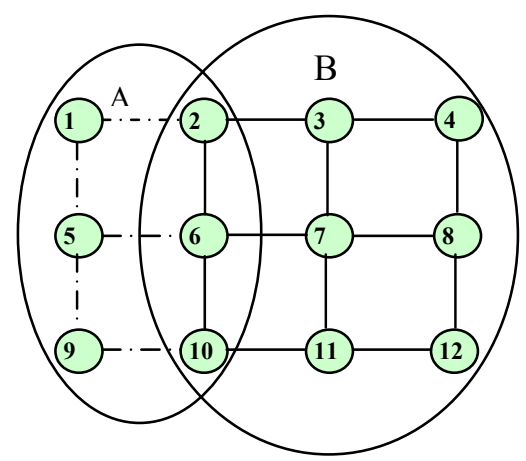

Figure 11.3 Subset A, B and C

Rosenthal did not provide a guideline on how to separate the network and how to combine the subnetworks together. Wagner et al. (1988a) proposed to separate the network until each subnetwork only contained one link and combined each link successively to obtain the connectivity of the complete network; while Carlier and Lucet (1996) proposed to separate the subnetwork into nodes. Each subnetwork only contained one node, and nodes were merged progressively to schematize the entire network. With a looped system, the unequal relationship is always established, $|\mathrm{V}| \leq|\mathrm{E}|$ (the 
number of vertices is less than or equal to the number of links). It means fewer iterations are required to use the node approach. In this research, the latter approach to study connectivity problem is employed.

Several features are critical to the decomposition method, namely computational complexity, network representation; boundary set identification, and class enumeration. All information about the boundary set, the classes of the boundary set must be easily and directly accessible by the computer program. Network representation, boundary set identification and class enumeration, are all related with, and contribute to, computational complexity. In order to better understand the following three methods, the computational complexity problem is introduced.

\subsubsection{Computational Complexity Problem}

Rosenthal lists the total number of classes according to the size of boundary set as shown in Table 11.2. From Table 11.2, the total number of partitions increases exponentially with the size of boundary set. In order to reduce the number of classes, the size of the boundary sets must be as small as possible. In this study, the maximum boundary set is of 4 .

Table 11.2 Number of Partitions for Varying Boundary Size (Ref. Rosenthal, 1977)

\begin{tabular}{cccccccccc}
\hline $\begin{array}{c}\text { Boundary } \\
\text { Size }\end{array}$ & 2 & 3 & 4 & 5 & 6 & 7 & 8 & 9 & 10 \\
\hline $\begin{array}{c}\text { Number } \\
\text { of } \\
\text { Partitions }\end{array}$ & 2 & 5 & 15 & 52 & 203 & 877 & 4140 & 21147 & 115975 \\
\hline
\end{tabular}

The rationale to set the maximum size to be 4 is that a junction node in a distribution system normally does not have more than 4 links to which it is connected. This means that the size of boundary set would not exceed 4 and the partition of the boundary set would not exceed 15, allowing the decomposition method to remain practicable. As stated later, we also develop a method, 'Boundary Nodes Identification Method', to efficiently reduce the boundary size. If a larger boundary set than 4 is encountered, the computer program will stop the iteration procedure automatically and switch to the factoring and reduction procedure as introduced previously.

\section{Graph Presentation}

To make programming feasible, the graph is represented so that the information is easy to access. In this sense, the adjacency list is used to 
represent a graph. Since $G=(V, E), V$ is the entire set of vertices as stated previously, and a vertex array is used to represent V. Each element in this array contains an adjacency list, which is an array of nodes.

Table 11.3 shows the adjacency list representation of the complete graph (left part) illustrated in Figure 11.3

Table 11.3 Adjacency List Representation

\begin{tabular}{cc}
\hline Node & Adjacency List \\
\hline 1 & 2,5 \\
2 & $1,3,6$ \\
3 & $2,4,7$ \\
4 & 3,8 \\
5 & $1,6,9$ \\
6 & $2,5,7,10$ \\
7 & $3,6,8,11$ \\
8 & $4,7,12$ \\
9 & 5,10 \\
10 & $6,9,11$ \\
11 & $7,10,12$ \\
12 & 8,11 \\
\hline
\end{tabular}

\section{Boundary Node Identification}

To make the boundary set as small as possible, a procedure to identify boundary nodes is developed. Taking the graph shown in Figure 11.3 as an example to illustrate the procedure:

1) Add 1,2 to $\mathrm{A}, \mathrm{A}=\{1,2\}$

2) Cancel 1 and 2 from the adjacency lists of each other, $1=\{5\}$, and $2=\{3,6\}$. The boundary set $C=\{1,2\}$

3) Compare the adjacency lists of 1 and 2, choose the smallest adjacency list. Currently, the size of 1 is 1 and the size of 2 is 2 , then choose one node from 1's adjacency list, e.g. 5, $A=\{1,2,5\}$

4) If there are at least two nodes in one adjacency set, the total number of all possible nodes to be introduced into A, shall be computed, and 
choose the one with the least number of total adjacent nodes.

5) Cancel 1 from 5's adjacency list and cancel 5 from 1's adjacency list, $1=\{\}, 2=\{3,6\}$ and $5=\{6,9\}$, the boundary set $C=\{2,5\}$ because the adjacency list of 1 is empty now.

6) Repeat 3) and 5) until all the nodes have been added into A

In this example, the maximum boundary set is 4 by following this procedure.

\section{Class Enumeration}

In Table 11.4, we list the partitions of each boundary set and the formulas to calculate the probability of each class. The probability of each subnetwork is the sum of the probabilities of each class.

Table 11.4 Class enumeration.

\begin{tabular}{|c|c|c|c|c|}
\hline $\begin{array}{l}\text { Boundary } \\
\text { size }\end{array}$ & Node & $\begin{array}{c}\text { Partition } \\
\text { Representation }\end{array}$ & Blocks & Formulas \\
\hline \multirow[t]{2}{*}{2} & $\mathrm{a}, \mathrm{b}$ & {$[\mathrm{a}, \mathrm{b}]$} & 1 & $\left(1-q_{a} q_{b}\right) P_{k-1,1}$ \\
\hline & & {$[\mathrm{a}][\mathrm{b}]$} & 2 & $\mathrm{p}_{\mathrm{a}} \mathrm{p}_{\mathrm{b}} \operatorname{Pr}_{\mathrm{k}-1,2}$ \\
\hline \multirow[t]{5}{*}{3} & $\mathrm{a}, \mathrm{b}, \mathrm{c}$ & {$[a, b, c]$} & 1 & $\left(1-q_{a} q_{b} q_{c}\right) \operatorname{Pr}_{k-1,1}$ \\
\hline & & {$[a, b][c]$} & 2 & $\left(1-q_{a} q_{b}\right) q_{c} \operatorname{Pr}_{k-1,21}$ \\
\hline & & {$[a, c][b]$} & & $\left(1-q_{a} q_{c}\right) q_{b} \operatorname{Pr}_{k-1,22}$ \\
\hline & & {$[\mathrm{b}, \mathrm{c}][\mathrm{a}]$} & & $\left(1-q_{a} q_{b}\right) q_{c} \operatorname{Pr}_{k-1,23}$ \\
\hline & & {$[\mathrm{a}][\mathrm{b}][\mathrm{c}]$} & 3 & $p_{a} p_{b} p_{c} p_{d} P_{k-1,3}$ \\
\hline \multirow[t]{15}{*}{4} & $\mathrm{a}, \mathrm{b}, \mathrm{c}, \mathrm{d}$ & {$[\mathrm{a}, \mathrm{b}, \mathrm{c}, \mathrm{d}]$} & 1 & $\left(1-q_{a} q_{b} q_{c} q_{d}\right) \operatorname{Pr}_{k-1,1}$ \\
\hline & & {$[\mathrm{a}, \mathrm{b}][\mathrm{c}, \mathrm{d}]$} & 2 & $\left(1-q_{a} q_{b}\right)\left(1-q_{c} q_{d}\right) \operatorname{Pr}_{k-1}$, \\
\hline & & {$[a, c][b, d]$} & & $\left(1-q_{a} q_{b}\right)\left(1-q_{c} q_{d}\right) \operatorname{Pr}_{k-1,}$ \\
\hline & & {$[\mathrm{a}, \mathrm{d}][\mathrm{b}, \mathrm{c}]$} & & 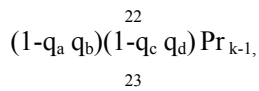 \\
\hline & & {$[\mathrm{a}][\mathrm{b}, \mathrm{c}, \mathrm{d}]$} & & $p_{a}\left(1-q_{b} q_{c} q_{d}\right) \operatorname{Pr}_{k-1,24}$ \\
\hline & & {$[\mathrm{b}][\mathrm{a}, \mathrm{c}, \mathrm{d}]$} & & $\mathrm{p}_{\mathrm{b}}\left(1-\mathrm{q}_{\mathrm{a}} \mathrm{q}_{\mathrm{c}} \mathrm{q}_{\mathrm{d}}\right) \operatorname{Pr} \mathrm{r}_{\mathrm{k}-1,25}$ \\
\hline & & {$[\mathrm{c}][\mathrm{a}, \mathrm{b}, \mathrm{d}]$} & & $\mathrm{p}_{\mathrm{c}}\left(1-\mathrm{q}_{\mathrm{a}} \mathrm{q}_{\mathrm{b}} \mathrm{q}_{\mathrm{d}}\right) \operatorname{Pr}_{\mathrm{k}-1,26}$ \\
\hline & & {$[d][a, b, c]$} & & $\mathrm{p}_{\mathrm{d}}\left(1-\mathrm{q}_{\mathrm{a}} \mathrm{q}_{\mathrm{b}} \mathrm{q}_{\mathrm{c}}\right) \operatorname{Pr}_{\mathrm{k}-1,27}$ \\
\hline & & {$[\mathrm{a}][\mathrm{b}, \mathrm{c}][\mathrm{d}]$} & 3 & $p_{a}\left(1-q_{b} q_{c}\right) p_{d} \operatorname{Pr}_{k-1,31}$ \\
\hline & & {$[\mathrm{a}][\mathrm{b}][\mathrm{c}, \mathrm{d}]$} & & $p_{a}\left(1-q_{c} q_{d}\right) p_{b} \operatorname{Pr}_{k-1,32}$ \\
\hline & & {$[\mathrm{a}][\mathrm{c}][\mathrm{b}, \mathrm{d}]$} & & $p_{a}\left(1-q_{b} q_{d}\right) p_{c} \operatorname{Pr}_{k-1,33}$ \\
\hline & & {$[\mathrm{b}][\mathrm{a}, \mathrm{c}][\mathrm{d}]$} & & $\mathrm{p}_{\mathrm{b}}\left(1-\mathrm{q}_{\mathrm{a}} \mathrm{q}_{\mathrm{c}}\right) \mathrm{p}_{\mathrm{d}} \operatorname{Pr}_{\mathrm{k}-1,34}$ \\
\hline & & {$[\mathrm{b}][\mathrm{a}, \mathrm{d}][\mathrm{c}]$} & & $\mathrm{p}_{\mathrm{b}}\left(1-\mathrm{q}_{\mathrm{a}} \mathrm{q}_{\mathrm{d}}\right) \mathrm{p}_{\mathrm{c}} \operatorname{Pr}_{\mathrm{k}-1,35}$ \\
\hline & & {$[\mathrm{c}][\mathrm{d}][\mathrm{a}, \mathrm{b}]$} & & $\mathrm{p}_{\mathrm{c}}\left(1-\mathrm{q}_{\mathrm{a}} \mathrm{q}_{\mathrm{b}}\right) \mathrm{p}_{\mathrm{d}} \operatorname{Pr}_{\mathrm{k}-1,36}$ \\
\hline & & {$[\mathrm{a}][\mathrm{b}][\mathrm{c}][\mathrm{d}]$} & 4 & $\mathrm{p}_{\mathrm{a}} \mathrm{p}_{\mathrm{b}} \mathrm{p}_{\mathrm{c}} \mathrm{p}_{\mathrm{d}} \mathrm{Pr}_{\mathrm{k}-1,4}$ \\
\hline
\end{tabular}


In this table, a, b, c, d, e represent the nodes in the boundary set. As stated earlier, $p_{a}$ represents the reliability of the link connecting the nodes inside the boundary set and the node outside, which is going to add to the subgraph. $\mathrm{q}_{\mathrm{a}}$ is the failure rate of this link, $\mathrm{q}_{\mathrm{a}}=1-\mathrm{p}_{\mathrm{a}}$. Similarly, $\mathrm{p}_{\mathrm{b}}, \mathrm{p}_{\mathrm{c}}, \mathrm{p}_{\mathrm{d}}$ and $\mathrm{q}_{\mathrm{a}}, \mathrm{q}_{\mathrm{b}}, \mathrm{q}_{\mathrm{c}}, \mathrm{q}_{\mathrm{d}}$, represent the reliabilities and failure rates respectively. $\operatorname{Pr}_{\mathrm{k}-1, \mathrm{n}}$ $\ldots$ is the probability corresponding to a certain class. From this table, we can see the importance to identify each class and to make the classes easily accessible.

\subsection{Computer Program and Case Studies}

In order to perform both the analytical approach and the simulation approach, a computer program was developed by implementing the EPANET Toolkits. The system diagram is shown in Figure 11.4.

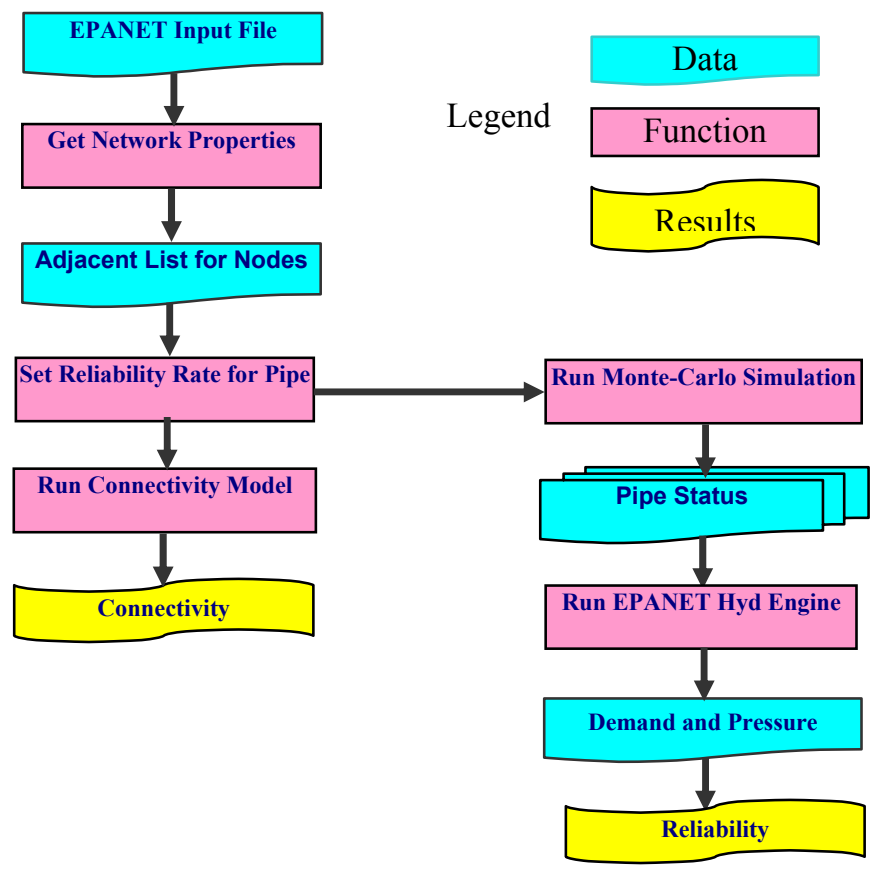

Figure 11.4. System Diagram 
The computer program first loads the EPANET input data file and obtains the network properties by parsing the input file. Once the links and nodes are given, the adjacency list for each node can be developed. A representation of the network is thus achieved. After the reliability rate of each link is set, the command to run the connectivity model or simulation model will be issued. The results will be either written to an Excel file or show on the result window as shown in Figure 11.5.

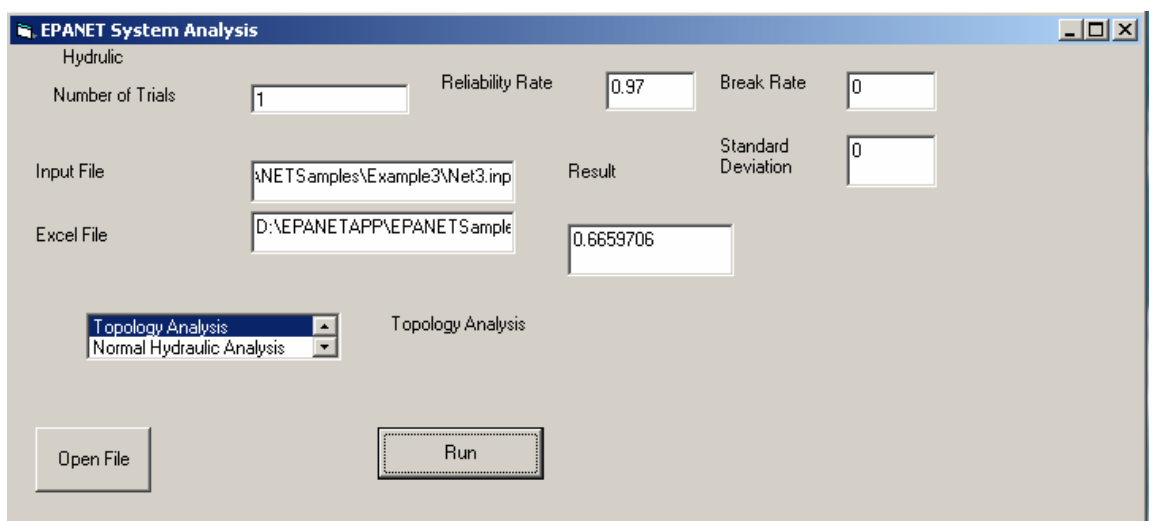

Figure 11.5. User Interface

\subsubsection{Simulation Approach}

Two case studies were conducted in this research. Case 1 is example 2 of EPANET and Case 2 is example 3 of EPANET.

As shown in Figure 11.6, the system demonstrated in Case 1 consists of 40 pipes, 36 supply nodes and one source, one pump station, and one tank. This system is a simple series-parallel reducible network and the computations can be performed by simply using the factoring and reduction method.

Case 2 is more complicated than Case 1. As shown in Figure 11.7, the system of Case 2 consists of 119 pipes, 97 supply nodes, two sources, three elevated tanks, and two pumps.

Reliability rate is set to be 0.97 for each link in this study. The failure rate of each link is thus to be $1-0.97=0.03$. In order to perform simulation approach, the minimum and maximum pressure and base demand for each supply nodes is specified in Table 11.5. 


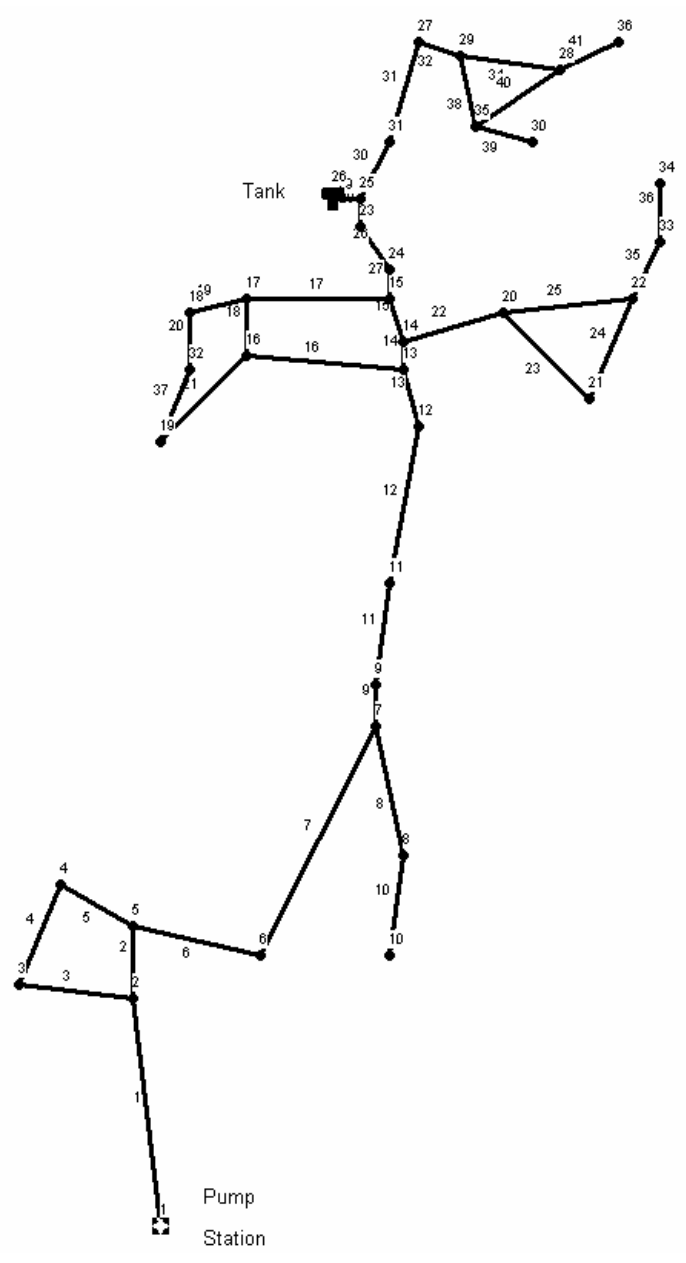

Figure 11.6 Cases 1(Example 2 of EPANET) 


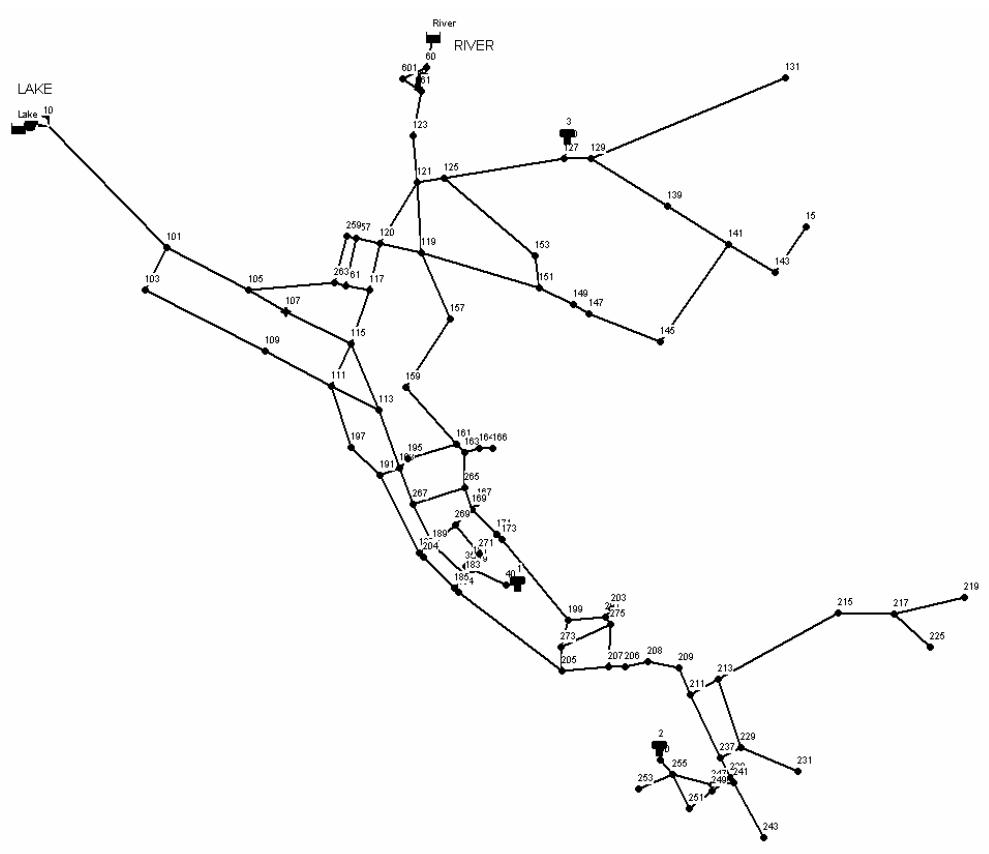

Figure 11.7 Case 2 (Example 3 in EPANET)

Table 11.5 Parameter Setting

Parameters Maximum Pressure (psi) Minimum Pressure (psi) Base Demand (cfs)

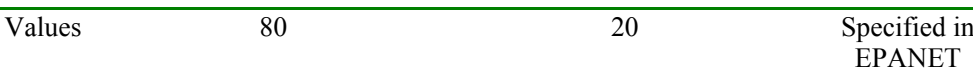

Input File

Table 11.6 shows the reliability using the simulation approach at $95 \%$ confidence level. As explained earlier, the number of the possible configurations of the system is $2^{\mathrm{N}}$ where $\mathrm{N}$ is number of the links. When the system is large, the number of possible configurations becomes extremely large. Knowing that some trials might be identical, 1000 trials only cover a very small portion of the whole configurations. However, to use simulation approach, 1000 trials takes $153 \mathrm{~s}$ and $340 \mathrm{~s}$ for Case 1 and Case 2 respectively. In many circumstances, it is not affordable to run a large 
number of trials. The simulation results are an average of the 1000 trials, the question remains whether the results can be trusted as representative of the true reliability of the system. To answer this question, we need to introduce statistics in the simulation approach. Because the population of the configurations is extremely large, the population can be treated as infinite. The simulation approach thus becomes a simple random replacement sampling problem in statistics. After the trials, the standard deviation for the number of trials can be obtained, and thus statistical measures can be used to estimate the reliability value. If the confidence interval satisfies the accuracy requirement, we may be able to trust the result. If the confidence interval is too broad, more trials may need to be completed before the accuracy requirement is met.

Table 11.6 Simulation results.

\begin{tabular}{|c|c|c|c|c|}
\hline \multirow[t]{2}{*}{$\begin{array}{l}\text { Measurem } \\
\text { ent }\end{array}$} & \multicolumn{2}{|c|}{$\begin{array}{l}\text { Example } 2 \text { (1000 Trials) } \\
\text { Nodes: } 36 \\
\text { Links: } 40\end{array}$} & \multicolumn{2}{|c|}{$\begin{array}{l}\text { Example } 3 \text { (1000 Trials) } \\
\text { Nodes: } 97 \\
\text { Links: } 119\end{array}$} \\
\hline & Demand & Pressure & Demand & Pressure \\
\hline Reliability & $0.86 \pm 0.02$ & $0.86 \pm 0.02$ & $0.97 \pm 0.002$ & $0.91 \pm 0.02$ \\
\hline Time (sec) & \multicolumn{2}{|c|}{153} & \multicolumn{2}{|c|}{340} \\
\hline
\end{tabular}

\subsubsection{Analytical Approach}

To examine the applicability of the analytical methods, we first conducted a test using the two very simple networks taken from the paper of Wagner et al. (1998a). Network A consists of 10 nodes and 12 links as shown in Figure 11.6. Network B consists of 10 nodes and 14 links as shown in Figure 11.7.

Note that node 90 of network B is surrounded by 5 nodes. It is a typical irreducible network. However, to use the boundary set identification method developed in this study, the maximum boundary set is 3 . It provides additional evidence that the maximum boundary set of 4 is a practicable approach. To solve the two simple networks on a CPU 2.8 Hz P6 Computer, the total computation time for network A and network B is $0.34 \mathrm{~s}$ and $0.45 \mathrm{~s}$, respectively. 


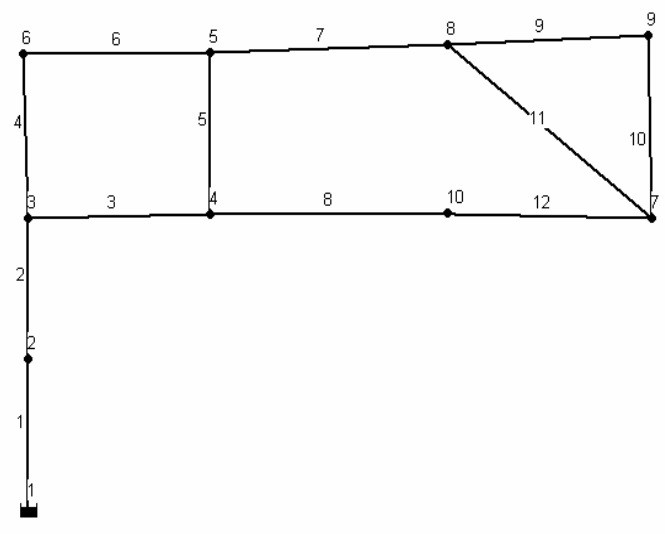

Figure 11.6. Network A in Wagner et al. (1988a).

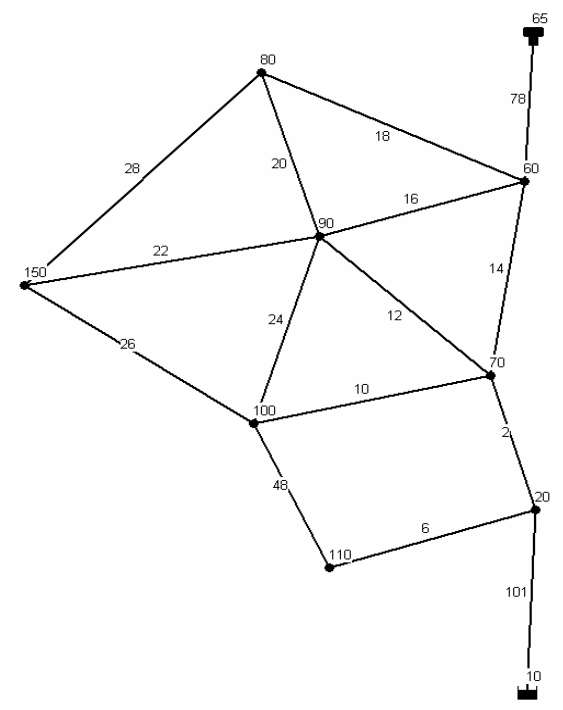

Figure 11.7. Network B in Wagner et al. (1988a).

We also conducted case studies on two real networks: Cases 1 and 2. Case 1 as shown in Figure 11.6 is a simple series-parallel reducible network 
and the computation can be performed by simply using the factoring and reduction method. Case 2, as shown in Figure 11.7, is a more complicated network. Many loops are present in the network making this an irreducible network. The decomposition method has to be applied to achieve the connectivity. For this network, the maximum boundary size is 3 .

Table 11.7 shows the computational results. The reliability is represented by connectivity in the analytical approach. All links' reliability rate is set to be 0.97 .

The reliabilities of Networks A and B are much larger than the reliabilities of Cases 1 and 2. This occurs because the numbers of nodes of Network A and Network B are much less than Case 1 and Case 2, although Case 2 also has an extensive redundancy. This means that the connectivity depends on the number of nodes of a system. Case 2 has two sources and three tanks in the system.

Table 11.7 Computational result.

\begin{tabular}{|c|c|c|c|c|}
\hline Networks & $\begin{array}{c}\text { Network } \\
\text { Properties }\end{array}$ & $\begin{array}{c}\text { Maximum } \\
\text { Boundary Size }\end{array}$ & Reliability & $\begin{array}{c}\text { Computation } \\
\text { Time }\end{array}$ \\
\hline Network A & $\begin{array}{c}10 \text { Nodes, } 10 \\
\text { Links }\end{array}$ & 2 & 0.94 & $0.34 \mathrm{~s}$ \\
\hline Network B & $\begin{array}{l}10 \text { Nodes, } 15 \\
\text { Links }\end{array}$ & 3 & 0.94 & $0.45 \mathrm{~s}$ \\
\hline Case 1 & $\begin{array}{l}36 \text { Nodes, } 40 \\
\text { Links }\end{array}$ & 2 & 0.56 & $0.62 \mathrm{~s}$ \\
\hline Case 2 & $\begin{array}{c}97 \text { Nodes, } 119 \\
\text { Links }\end{array}$ & 3 & 0.67 & $4.6 \mathrm{~s}$ \\
\hline
\end{tabular}

However, the connectivity approach treats the source nodes as regular nodes in the connectivity study. Additionally, even if it may have minimal effect on the water supply to the whole system, when a leaf node is isolated, the connectivity approach identifies a failure event under this circumstance. The connectivity approach thus cannot truly reflect the unique characteristics of a water distribution system. In this case, the simulation approach may need to be used.

\subsection{Discussion and Conclusion}

The interpretation and utilization of the connectivity indicator is a major issue. Since the size and roughness of pipes may vary significantly over a system, and nodes are modeled to be perfectly reliable, the connectivity can be considered as an upper bound reliability of the studied system (Wagner et 
al., 1988a). However, in reality, one node being isolated from a system may not cause significant consequence unless the nodal demand or importance is extremely high, or this node is located upstream of many nodes and its isolation results in the isolation of other nodes. The disadvantage limits the use of connectivity as a reliability indicator and the connectivity indicator has to couple with other measurements to generate a good characterization of a system. This explains why the reliability obtained from the simulation approaches is larger than the value obtained from the analytical approaches in the two case studies. A very important advantage of simulation approaches over analytical techniques is that simulation approaches permit the use of any reliability measure that can be derived from the hydraulic performance.

Table 11.8 Comparing time for two approaches.

\begin{tabular}{ccc}
\hline Alternative Approach & $\begin{array}{c}\text { Time }(\mathrm{sec}) \\
\text { for Case 1 }\end{array}$ & $\begin{array}{c}\text { Time (sec) } \\
\text { for Case 2 }\end{array}$ \\
\hline Analytical & 1 & 4 \\
\hline $\begin{array}{c}\text { Simulation } \\
(1000 \text { trials })\end{array}$ & 153 & 340 \\
\hline
\end{tabular}

Monte-Carlo simulation is often employed in the simulation approach. This approach usually involves large computational cost. Table 11.8 compares the time involved in the two approaches. It is obvious that the simulation approach consumes much longer times than the analytical approach. To examine the performance of an existing system, the computation time of the simulation approach might be affordable. However, to conduct system design optimization, the reliability results may require much more significant computing to evaluate hundreds and thousands of design alternatives in order to achieve an optimized solution. In these cases, the simulation approach probably is not an affordable and practicable approach. Under this circumstance, a more feasible approach is to employ the analytical approach during the optimization process and to evaluate the final solution by using the simulation approach.

In conclusion, although the analytical approach addresses the redundancy issue effectively and provides insight into the water distribution system, it only provides a static view of reliability in terms of topology, lacking acceptable coverage of hydraulic aspects and water quality aspects. In contrast, the simulation approach enables investigations with real world 
scenarios as well as the consequence of mechanical failure in terms of pressure and demand, generating more detailed reliability pictures about water distribution systems. The major shortcoming of the simulation approach is its lengthy computational requirements. As the size of the water distribution system under estimation increases, more trials may be required to achieve a stable and trusted reliability result. If the computation time problem cannot be conquered, the simulation approach may not be a practical approach under many circumstances. This research suggests that the selection of the simulation approach and analytical approach mainly depends on the evaluation purpose. In many circumstances, the two approaches should be integrated and used alternatively in different phases of reliability evaluations.

\section{Acknowledgment}

Appreciation is expressed to Dr. Rosenthal for his assistance in clarifying some principles of decomposition method. As well, thanks are also extended to Dr. Rossman for assistance in recompiling the EPANET Toolkits.

\section{References}

Babovic, Vladan; Drecourt, Jean-Philippe; Keijzer, Maarten; Hansen, Peter Friss; (2002). "A data mining approach to modeling of water supply assets", Urban Water, 4 (2002), pp. 401-414

Carlier, Jacques; and Lucet, Corinne; (1996). "A decomposition algorithm for network reliability evaluation", Discrete Applied Mathematics, V65, 141-156

Cullinane, M. J. , Lansey, K. E., and Mays, L. W. (1992). "Optimization-availabilitybased design of water distribution networks ”, Journal of Hydraulic Engineering, 118(3), 420-441

Engelhardt, M. O., Skipworth, P. J., Savic, D. A., Saul, A. J., \& Walters, G. A. (2000). "Rehabilitation strategies for water distribution networks: a literature review with a UK perspective", Urban Water, 2(2), 153-170.

Goulter, Ian C; (1988). "Measures of Internal Redundancy in Water Distribution Network Layouts", Journal of Information and Optimization Science, 9:363-390

Goulter, Ian C; and Coals, A.; (1986). "Quantitative approaches to reliability assessment in pipe networks", J. Hydraul. Eng., 116(2), 211-229

Hobbs, B. F.; and Beim, G. K.; (1988). "Analytical simulation of water system capacity reliability”, Water Resources Research, 24(9). 1431-1444 
Hosoi, Y; and Kido, Y; (2001). "Water Supply Systems-Comparative Reliability Analysis of The Distribution Areas of A Water Supply System and Its Application for An Earthquake Risk Analysis", Water Science and Technology-Water Supply, v. $1, n .4$ p. 217,10 p

Huang, J., E. McBean and W. James. 2004. "A Review of Reliability Analysis for Water Quality in Water Distribution Systems." Journal of Water Management Modeling R223-07. doi: 10.14796/JWMM.R223-07.

Jacobs, P., and Goulter, Ian C.; (1988). Evaluation of Methods for Decomposition of Water Distribution Networks for Reliability Analysis, Civil Engng Systems, 5: 58-64

Kalungi, Paul; Tanyimboh, Tiku T.; (2003). "Redundancy model for water distribution systems", Reliability Engineering and System Safety, 82(2003), pp. 275-286

Kleiner, Yehuda (2001). "Scheduling Inspection and Renewal of Large Infrastructure Assets", Journal of Infrastructure Systems, ASCE, 7(4), 136-143

Mays, Larry W; (1996). "Review of reliability analysis of water distribution system", Stochastic Hydraulics, Proceedings of the Seventh IAHR International Symposium

Mays, Larry W; (2000). Chapter 18: Reliability Analysis for Design, AWWA, "Water Distribution Systems Handbook" Edited by Mays, Larry W., Publisher: New York; London: McGraw-Hill, c2000, ISBN:0071342133.

Ostfeld, Avi ; (2001). "Reliability analysis of regional water distribution systems", Urban Water, 3(2001), pp. 253-260

Ostfeld, Avi ; Kogan, Dimitri; Shamir, Uri; (2002). "Reliability simulation of water distribution systems - single and multiquality", Urban Water, 4(2002), pp. 53-61

Ostfeld, Avi; (2005). "Water Distribution Systems Connectivity Analysis", Journal of Water Resources Planning and Management. v. 131 , n. 1, p. 58-66

Rosenthal Arnie; (1977). "Computing the reliability of complex networks", SIAM J. APPL. MATH.

Rossman, Lewis A.; (2000). "EPANET2 Users Manuel"

Shinstine, Debbie S.; Ahmed, Iffekhar; and Lansey, Kevin E.; (2002). "Reliability/ Availability Analysis of Municipal Water Distribution Networks: Case Study", Journal of Water Resources Planning and Management. v. 128 , n. 2 , p. 140 , 12 p

Su, Y.; Mays, L.; Duan, N.; and Lansey,K.; (1987). "Reliability Based Optimization for Water Distribution Systems", ASCE Journal of Hydraulic Engineering, 113:589-596

Tanyimboh, T. T.; Burd, R.; Burrows, R.; Tabesh, M.; (1999), "Modelling and reliability analysis of water distribution systems", Water Science and Technology Volume: 39, Issue: 4, pp. 249-255

Tolson, Bryan; Maier, Holger R.; Simpson, Angus R.; and Lence, Barbara J.; (2004). "Genetic Algorithms for Reliability-Based Optimization of Water Distribution Systems", J of Water Resources Planning and Management. v. 130, n. 1, pp. 63-72

Wagner, J., U. Shamir, and D. Marks, (1988a). Water Distribution Reliability Analytical Methods, ASCE J. Water Resources Planning and Management, 114:253-275

Wagner, J., U. Shamir, and D. Marks, (1988b). "Water Distribution Reliability Simulation Methods", ASCE J.Water Resources Planning and Management, 114:276-294

Yang, Shu-li; Hsu, Nien-Sheng; Louie, Peter W. F.; Yeh, William, W-G; (1996). "Water Distribution Network Reliability: Connectivity Analysis", Journal of Infrastructure Systems, 2(2), 54-64 
Xu, Chengchao and Goulter, Ian C; (1998). "Probabilistic Model for Water Distribution Reliability", Journal of Water Resources Planning and Management. v. 124 , n. 4 , p. $218,12 \mathrm{p}$ 